\title{
Immunoreactivities of calbindin-D28k, calretinin and parvalbumin in the somatosensory cortex of rodents during normal aging
}

\author{
JI HYEON AHN ${ }^{1 *}$, SEONGKWEON HONG ${ }^{2 *}$, JOON HA PARK $^{1}$, IN HYE KIM ${ }^{3}$, \\ JEONG HWI CHO ${ }^{3}$, TAE-KYEONG LEE ${ }^{3}$, JAE-CHUL LEE ${ }^{3}$, BAI HUI CHEN ${ }^{4}$, BICH-NA SHIN ${ }^{5}$, \\ EUN JOO BAE ${ }^{6}$, YONG HWAN JEON ${ }^{7}$, YOUNG-MYEONG KIM ${ }^{8}$, MOO-HO WON $^{3}$ and SOO YOUNG CHOI ${ }^{1}$
}

\author{
${ }^{1}$ Department of Biomedical Science and Research Institute for Bioscience and Biotechnology, Hallym University, Chuncheon, \\ Gangwon 24252; Departments of ${ }^{2}$ Surgery and ${ }^{3}$ Neurobiology, School of Medicine, Kangwon National University, \\ Chuncheon, Gangwon 24341, Republic of Korea; ${ }^{4}$ Department of Histology and Embryology, \\ Institute of Neuroscience, Wenzhou Medical University, Wenzhou, Zhejiang 325035, P.R. China; \\ ${ }^{5}$ Department of Physiology, College of Medicine, Hallym University, Chuncheon, Gangwon 24252; ${ }^{6}$ Department of Pediatrics, \\ Chuncheon Sacred Heart Hospital, College of Medicine, Hallym University, Chuncheon, Gangwon 24253; \\ ${ }^{7}$ Department of Radiology, School of Medicine, Kangwon National University, Chuncheon, \\ Gangwon 24289; ${ }^{8}$ Department of Molecular and Cellular Biochemistry, School of Medicine, \\ Kangwon National University, Chuncheon, Gangwon 24341, Republic of Korea
}

Received August 11, 2016; Accepted March 1, 2017

DOI: $10.3892 / \mathrm{mmr} .2017 .7573$

\begin{abstract}
Calbindin-D28k (CB), calretinin (CR) and parvalbumin (PV), which regulate cytosolic free $\mathrm{Ca}^{2+}$ concentrations in neurons, are chemically expressed in $\gamma$-aminobutyric acid (GABA)ergic neurons that regulate the degree of glutamatergic excitation and output of projection neurons. The present study investigated age-associated differences in $\mathrm{CB}, \mathrm{CR}$ and $\mathrm{PV}$ immunoreactivities in the somatosensory cortex in three species (mice, rats and gerbils) of young (1 month), adult (6 months) and aged (24 months) rodents, using immunohistochemistry and western blotting. Abundant CB-immunoreactive neurons were distributed in layers II and III, and age-associated alterations in their number were different according to the species. CR-immunoreactive
\end{abstract}

Correspondence to: Professor Moo-Ho Won, Department of Neurobiology, School of Medicine, Kangwon National University, 1 Kangwondaehak, Chuncheon, Gangwon 24341, Republic of Korea E-mail: mhwon@kangwon.ac.kr

Professor Soo Young Choi, Department of Biomedical Science and Research Institute of Bioscience and Biotechnology, Hallym University, 1 Hallymdaehak, Chuncheon, Gangwon 24252, Republic of Korea

E-mail: sychoi@hallym.ac.kr

*Contributed equally

Key words: somatosensory cortex, rodents, GABAergic neurons, calbindin-D28K, calretinin, parvalbumin neurons were not abundant in all layers; however, the number of CR-immunoreactive neurons was the highest in all adult species. Many PV-immunoreactive neurons were identified in all layers, particularly in layers II and III, and they increased in all layers with age in all species. The present study demonstrated that the distribution pattern of CB-, CR- and $\mathrm{PV}$-containing neurons in the somatosensory cortex were apparently altered in number with normal aging, and that $\mathrm{CB}$ and $\mathrm{CR}$ exhibited a tendency to decrease in aged rodents, whereas PV tended to increase with age. These results indicate that $\mathrm{CB}, \mathrm{CR}$ and $\mathrm{PV}$ are markedly altered in the somatosensory cortex, and this change may be associated with normal aging. These findings may aid the elucidation of the mechanisms of aging and geriatric disease.

\section{Introduction}

The somatosensory cortex, which is a part of the neocortex, receives the majority of general sensory signals for interpretation. The mammalian somatosensory cortex is divided into six layers containing many types of neurons, which have different functions. The superficial layers (layers I, II/III and IV), considered to be the most integrative layers, collect sensory information and distribute outputs to other cortical areas (1). The deeper layers (layers V and VI), which are output layers of the neocortex, innervate their associated cortical areas and subcortical nuclei (1).

The neocortex contains glutamatergic pyramidal cells and $\gamma$-aminobutyric acid (GABA)ergic non-pyramidal cells $(2,3)$, and GABAergic neurons constitute 20 to $30 \%$ of neurons (4). GABAergic neurons suppress and modulate pyramidal cell activities, and maintain the balance between excitation and 
inhibition, which is crucial for normal brain function (5). GABAergic neurons are divided into subtypes depending on their morphological, electrophysiological and chemical characteristics (3). Calbindin-D28k (CB)-, calretinin (CR)- and parvalbumin (PV)-containing neurons are chemically distinct subgroups of GABAergic interneurons, and the proteins are known as high-affinity cytosolic calcium $\left(\mathrm{Ca}^{2+}\right)$ binding proteins (CBPs) that regulate cytosolic free $\mathrm{Ca}^{2+}$ concentrations within neurons $(6,7) . \mathrm{Ca}^{2+}$ signaling is crucial in neuronal functions, such as neurotransmitter release and neuronal membrane excitability (8); therefore, altered regulation of intracellular $\mathrm{Ca}^{2+}$ concentration may be a major cause of brain aging or neuronal damage/death in ischemic insults and neurodegenerative diseases, including Parkinson's and Alzheimer's disease (9-11).

Previous studies have revealed the age-associated alterations in CBPs in various brain regions, including the hippocampus, striatum and cerebellum $(12,13)$. In addition, certain studies have reported the distribution and morphological characteristics of CBP-containing neurons in the cerebral cortex of mice $(14)$, rats $(3,15)$ and humans $(16)$. However, there are few studies regarding the age-associated alterations in CBP-containing neurons in the somatosensory cortex of mice, rats and gerbils. Therefore, the aim of the present study was to compare age-associated alterations in the expression levels of three CBPs (CB, CR and PV) in the somatosensory cortex between mice, rats and gerbils, which are useful animal models for evaluating aging (17-20).

\section{Materials and methods}

Experimental animals. Male ICR mice $(\mathrm{n}=36)$ and Sprague Dawley rats $(n=21)$ were purchased from Orient Bio Inc. (Seongnam, South Korea), and male Mongolian gerbils (Meriones unguiculatus, $\mathrm{n}=21$ ) were obtained from the Experimental Animal Center, Kangwon National University (Chuncheon, South Korea). The animals were used at postnatal month (PM) 1 (mice 13-15 g, rats 100-130 g and gerbils 25-30 g), PM 6 (mice 25-27 g, rats 400-450 g and gerbils 65-75 g) and PM 24 (mice 27-30 g, rats 600-700 g and gerbils 85-95 g), and defined as young, adult and aged, respectively, as the average lifespan of the three species is similar (21-23). The animals were housed in a conventional condition under an adequate temperature $\left(23 \pm 3^{\circ} \mathrm{C}\right)$ and relative humidity $(55 \pm 5 \%)$ with a $12 \mathrm{~h}$ light:dark cycle and were allowed free access to food and water. The animals were used according to the guidelines that are in compliance with the current international laws and policies (Guide for the Care and Use of Laboratory Animals, The National Academies Press, 8th Edition, 2011) (24) and experiments were approved by the Institutional Animal Care and Use Committee at Kangwon National University (approval no. KW-130424-3).

Immunohistochemistry. To investigate the age-associated alterations in $\mathrm{CB}, \mathrm{CR}$ and $\mathrm{PV}$ immunoreactivity in the somatosensory cortex of the three types of rodents, immunohistochemical staining and quantitative analysis of immunohistochemical data were performed according to our previously study (25). Briefly, animals ( $\mathrm{n}=7$ at each age in each group) were anesthetized with pentobarbital sodium
(40 mg/kg; JW Pharmaceutical Co., Ltd., Seoul, South Korea) and perfused transcardially with $4 \%$ paraformaldehyde. Subsequently, the brain tissue samples were serially sliced into $30 \mu \mathrm{m}$ coronal sections. Rabbit anti-CB (MAB1778; 1:1,000; Chemicon International, Inc., Temecula, CA, USA), mouse anti-CR (MAB1568; 1:1,000; Chemicon International, Inc.) or rabbit anti-PV (AB9312; 1:1,000; Chemicon International, Inc.) primary antibodies were used to incubate the sections overnight at $4^{\circ} \mathrm{C}$. A negative control test was performed using preimmune serum instead of a primary antibody in order to establish the specificity of the immunostaining. The negative control resulted in the absence of immunoreactivity in any neurons.

According to anatomical landmarks corresponding to anterioposterior from +1.34 to $-0.94 \mathrm{~mm}$ (mouse brain atlas) (26), from +1.70 to $-1.40 \mathrm{~mm}$ (rat brain atlas) (27) and from +0.70 to $-0.90 \mathrm{~mm}$ (gerbil brain atlas) (28), seven sections with $120-\mu \mathrm{m}$ intervals per animal were selected to quantitatively analyze CB, CR and PV immunoreactivity. As previously described (29), digital images of the somatosensory cortex were captured using an AxioM1 light microscope (Carl Zeiss AG, Oberkochen, Germany) equipped with a digital camera (Axiocam; Carl Zeiss AG) connected to a PC monitor. CB-, $\mathrm{CR}$ - and PV-immunoreactive neurons were counted in a designated column (all layers) of the somatosensory cortex (300- $\mu \mathrm{m}$ width), which was sequentially moved from the cortical surface to the white matter using an image analyzing system (Optimas software version 6.5; Media Cybernetics, Inc., Rockville, MD, USA). Cell counts were obtained by averaging the counts from each animal. The mean number was calibrated as a percentage, with the young group defined as $100 \%$.

Western blot analysis. To compare CB, CR and PV levels in the somatosensory cortex according to age, mice ( $n=5$ per age, mice used only to minimize the size of experimental groups) were used. Western blot analysis was performed according to our previous study (25). Briefly, the tissue samples were homogenized in $50 \mathrm{mM}$ phosphate-buffered saline ( $\mathrm{pH}$ 7.4) containing $0.1 \mathrm{mM}$ ethylene glycol bis(2-aminoethyl ether)- $\mathrm{N}, \mathrm{N}, \mathrm{N}^{\prime}, \mathrm{N}^{\prime}$ tetraacetic acid $(\mathrm{pH} 8.0), 0.2 \%$ Nonidet $\mathrm{P}-40,10 \mathrm{mM}$ ethylendiamine tetraacetic acid ( $\mathrm{pH} 8.0)$, $15 \mathrm{mM}$ sodium pyrophosphate, $100 \mathrm{mM} \beta$-glycerophosphate, $50 \mathrm{mM} \mathrm{NaF}, 150 \mathrm{mM} \mathrm{NaCl}, 2 \mathrm{mM}$ sodium orthovanadate, $1 \mathrm{mM}$ phenylmethylsulfonyl fluoride and $1 \mathrm{mM}$ dithiothreitol (DTT). Following centrifugation (at $16,000 \times \mathrm{g}$ for $20 \mathrm{~min}$ at $4^{\circ} \mathrm{C}$ ), the protein level in the supernatants was determined using a Pierce Bicinchoninic Protein Assay kit (Thermo Fisher Scientific, Inc., Waltham, MA, USA) with bovine serum albumin serving as a standard according to the manufacturer's protocols. Aliquots containing $20 \mu \mathrm{g}$ total protein were boiled in loading buffer containing $150 \mathrm{mM}$ Tris ( $\mathrm{pH}$ 6.8), $3 \mathrm{mM}$ DTT, $6 \%$ SDS, $0.3 \%$ bromophenol blue and $30 \%$ glycerol. The aliquots containing $20 \mu \mathrm{g}$ total protein were then loaded onto a $12 \%$ polyacrylamide gel. Following electrophoresis, the proteins were transferred to nitrocellulose transfer membranes (Pall Corporation, Port Washington, NY, USA). The membranes were incubated with rabbit anti-CB (MAB1778; 1:1,000; Chemicon International, Inc.), rabbit mouse anti-CR (MAB1568; 1:1,000; Chemicon International, Inc.), rabbit anti-PV (AB9312; 1:1,000; 
Chemicon International, Inc.) or mouse anti- $\beta$-actin (A5316; 1:5,000; Sigma-Aldrich; Merck KGaA, Darmstadt, Germany) primary antibodies overnight at $4^{\circ} \mathrm{C}$. Following washing with PBST three times, the membranes were incubated with peroxidase-conjugated mouse anti-goat (sc-2354; dilution, 1:5,000) or mouse anti-rabbit (sc-2357; dilution, 1:5,000; both from Santa Cruz Biotechnology) for $1 \mathrm{~h}$ at room temperature. Western blot analysis was performed with three repetitions. The results of the western blot analysis were scanned, and densitometric analysis for the quantification of the bands was performed using ImageJ software version 1.49 (National Institutes of Health, Bethesda, MD, USA), which was used to count relative optical density (ROD). CB, CR and PV levels were normalized to that of $\beta$-actin, which served as an internal control protein. A ratio of the ROD was calibrated as a percentage, with the young group defined as $100 \%$.

Statistical analysis. The data are presented as the mean \pm standard error of the mean. Differences among the groups were statistically analyzed by one-way analysis of variance with a post hoc Bonferroni's multiple comparison test in order to elucidate age-associated differences among groups using SPSS software version 12.0 (SPSS, Inc., Chicago, IL, USA). $\mathrm{P}<0.05$ was considered to indicate a statistically significant difference.

\section{Results}

\section{$C B$ immunoreactivity}

Mice. Numerous CB-immunoreactive neurons were identified in the young mice in all of the layers (Fig. 1); however, the density of the CB-immunoreactive neurons was markedly higher in layers II and III when compared with the other layers (Fig. 1A and J). In the adult mice, the distribution pattern of CB-immunoreactive neurons was similar to that of the young mice; however, the percentage mean number of CB-immunoreactive neurons in all layers was significantly increased by $20 \%$ when compared with the young mice (Fig. 1B and J). In the aged mice, the percentage mean number of CB-immunoreactive neurons in all layers was significantly decreased by 30 and $50 \%$ when compared with the young and adult mice, respectively (Fig. 1C and J).

Rats. In rats, the distribution pattern of $\mathrm{CB}$ immunoreactivity was similar to that of the mice (Fig. 1D-F); however, the percentage mean number of $\mathrm{CB}$-immunoreactive neurons in all layers was different to that of mice (Fig. 1J). The percentage mean number of $\mathrm{CB}$-immunoreactive neurons in all layers of the adult rats was significantly decreased by $23 \%$ when compared with the young rats (Fig. 1E and J). In the aged rats, the percentage mean number of $\mathrm{CB}$-immunoreactive neurons in all layers was significantly decreased by 42 and $29 \%$ when compared with the young and adult rats, respectively (Fig. 1F and J).

Gerbils. The general distribution pattern of CB immunoreactivity in the gerbils was similar to that of the mice (Fig. 1G-J). In the adult gerbils, the percentage mean number of CB-immunoreactive neurons in all layers was significantly increased by $37 \%$ when compared with that of the young gerbils (Fig. $1 \mathrm{H}$ and $\mathrm{J}$ ). In the aged gerbils, the percentage mean number of CB-immunoreactive neurons in all layers was markedly decreased by $17 \%$ when compared with the adult gerbils, and it was significantly increased by $20 \%$ when compared with the young gerbils (Fig. 1I and J).

\section{CR immunoreactivity}

Mice. In the young mice, a few CR-immunoreactive neurons were identified throughout all layers (Fig. 2); the majority were observed in layers II and III, and were sparsely found in layers V and VI (Fig. 2A). In the adult mice, the percentage mean number of CR-immunoreactive neurons in all layers was significantly increased by $98 \%$ when compared with the young mice (Fig. 2B and J). In the aged mice, the percentage mean number of CR-immunoreactive neurons in all layers was significantly decreased by $29 \%$ when compared with the adult group; however, it was significantly increased by $69 \%$ when compared with the young mice (Fig. $2 \mathrm{C}$ and $\mathrm{J}$ ).

Rats. In the rats, the distribution pattern of CR immunoreactivity was similar to that of the mice (Fig. 2D-F). In the adult rats, the percentage mean number of CR-immunoreactive neurons increased significantly in all layers by $70 \%$ when compared with the young rats (Fig. $2 \mathrm{E}$ and J). In the aged rats, the percentage mean number of CR-immunoreactive neurons was significantly decreased by $35 \%$ when compared with the adult rats; however, it was significantly increased by $35 \%$ when compared with the young rats (Fig. 2F and J).

Gerbils. In the gerbils, CR-immunoreactive neurons were relatively few in number when compared with those in the mice and rats (data not shown). In the adult gerbils, the percentage mean number of CR-immunoreactive neurons in all layers was significantly increased by $60 \%$ when compared with the young gerbils (Fig. $2 \mathrm{H}$ and J). In the aged gerbils, CR immunoreactivity was weak and the percentage mean number of CR-immunoreactive neurons in all layers was significantly decreased by $70 \%$ when compared with the adult gerbils and was significantly decreased by $19 \%$ when compared with the young gerbils (Fig. 2I and J).

\section{PV immunoreactivity}

Mice. In the young mice, a number of PV-immunoreactive neurons were observed throughout all layers (Fig. 3). In the adult mice, $\mathrm{PV}$-immunoreactive neurons were more prevalent in layers $\mathrm{V}$ and VI, and the percentage mean number of PV-immunoreactive neurons in all layers was marginally increased by $9 \%$ when compared with the young mice (Fig. 3B and $J$ ). In the aged mice, the percentage mean number of PV-immunoreactive neurons in all layers was significantly increased by 35 and $26 \%$ compared with that in the young and adult mice, respectively (Fig. 3C and J).

Rats. The distribution pattern of PV-immunoreactive neurons in the rat group was similar to that of the mice (Fig. 3D-F). In the adult rats, the percentage mean number of PV-immunoreactive neurons in all layers was significantly increased by $38 \%$ when compared with that of the young rats and PV immunoreactivity was observed in the neuropil in layer III (Fig. 3E). In the aged rats, the percentage mean number of PV-immunoreactive 


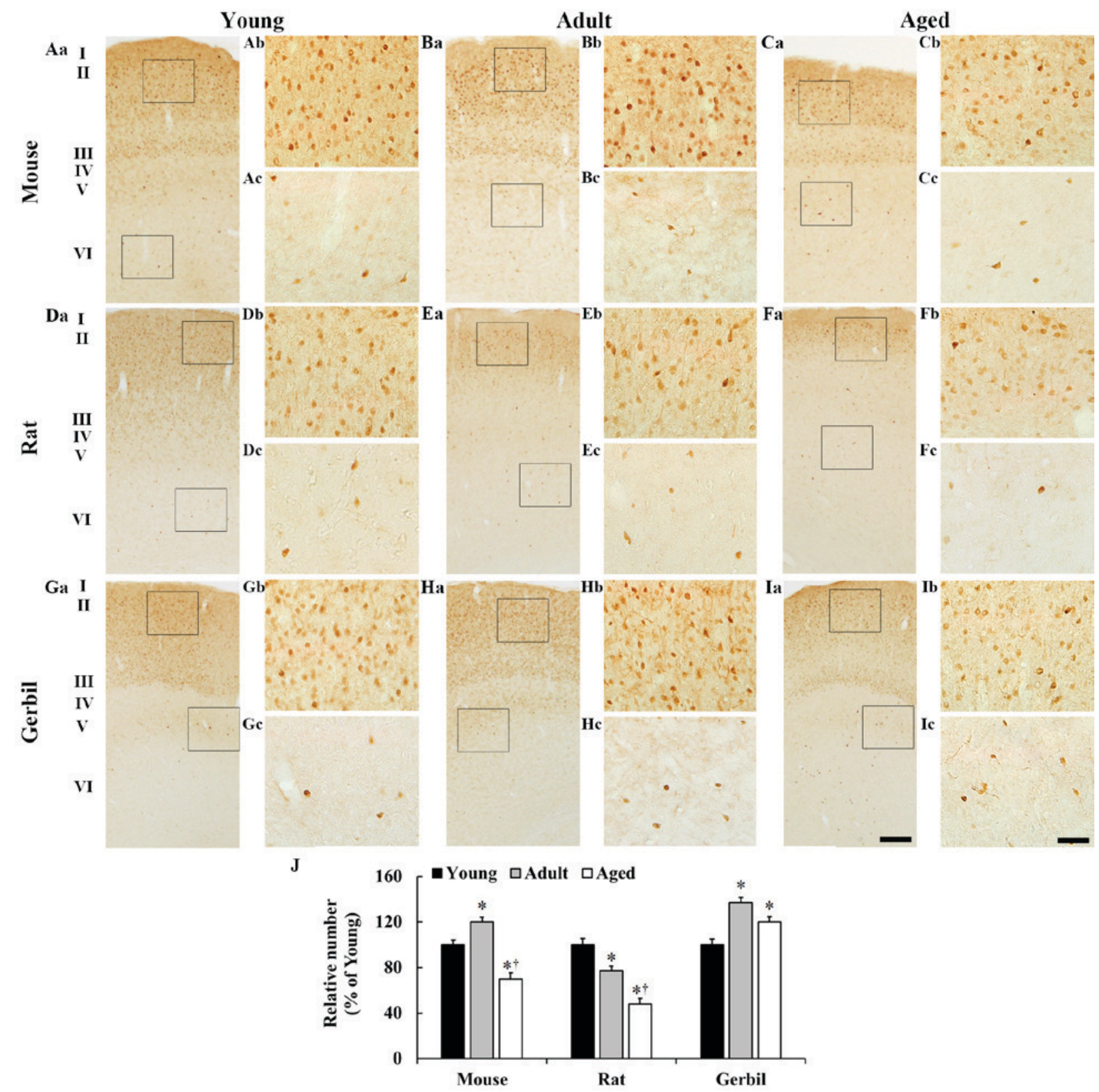

Figure 1. CB immunohistochemistry in the somatosensory cortex in the (A-C) mice, (D-F) rats and (G-I) gerbils. In all species, CB-immunoreactive neurons were primarily observed in layers II and III. The age-associated alterations were different amongst the species. The neuronal number in the aged was generally lower than that of the adults. Scale bars: (Aa-Ia), $200 \mu \mathrm{m}$ and (Ab-Ic), $25 \mu \mathrm{m}$. (J) The percentage mean number of CB-immunoreactive neurons in whole layers in the somatosensory cortex. ${ }^{*} \mathrm{P}<0.05$ vs. the young group; ${ }^{\dagger} \mathrm{P}<0.05$ vs. the adult group. Data are presented as the mean \pm the standard error of the mean ( $\mathrm{n}=7 /$ group). $\mathrm{CB}$, calbindin-D28k.

neurons in all layers was significantly increased by 23 and $61 \%$ when compared with the young and adult rats, respectively; however, PV immunoreactivity in the neuropil in layer III was similar to that of the adult rats (Fig. 3F and J).

Gerbils. In the young gerbils, the distribution pattern of PV-immunoreactive neurons was similar to that of the mice and rats (Fig. 3G-I); however, there were significantly fewer PV-immunoreactive neurons when compared with the numbers observed in the mice and rats (data not shown). In the adult gerbils, the percentage mean number of PV-immunoreactive neurons in all layers was significantly increased by $26 \%$ when compared with the young gerbils (Fig. 3G and J). In the aged gerbils, the percentage mean number of PV-immunoreactive neurons in all layers was increased by $14 \%$ when compared with the adult gerbils and significantly increased by $40 \%$ when compared with the young gerbils (Fig. 3I and J).

$C B, C R$ and $P V$ protein levels. Age-associated changes in the levels of $\mathrm{CB}, \mathrm{CR}$ and $\mathrm{PV}$ in the somatosensory cortex were examined in mice (Fig. 4). The altered pattern of each protein level was similar to the immunohistochemical results. The level of CB was greatest in the adult mice and lowest in the aged mice. Furthermore, the level of CR was greatest in the adult mice and lowest in the young mice. However, the level of PV was not significantly altered by age, although a marginal increase was observed with increased age. 


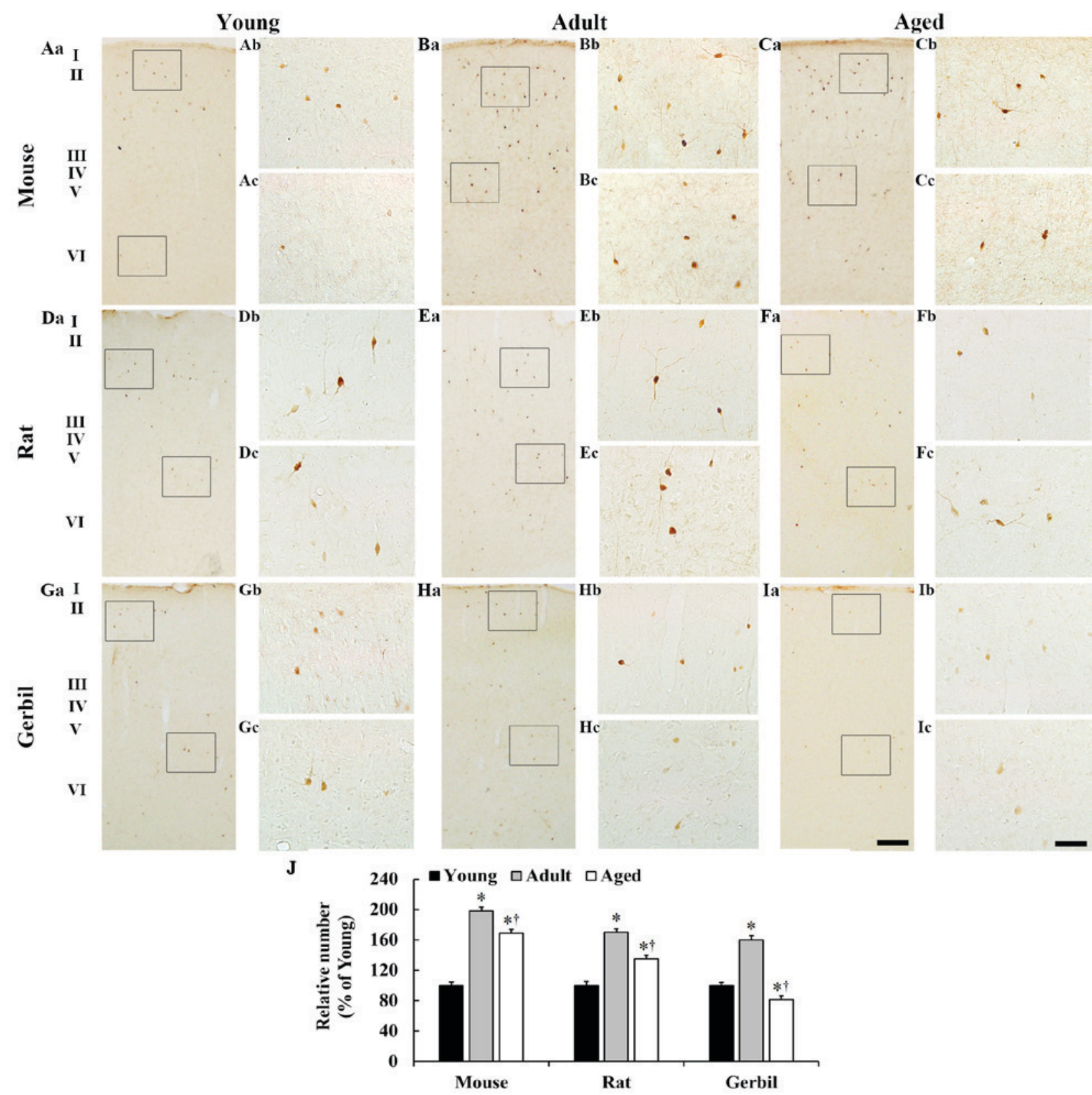

Figure 2. CR immunohistochemistry in the somatosensory cortex in the (A-C) mice, (D-F) rats and (G-I) gerbils. In all species, CR-immunoreactive neurons were primarily observed in layers II and III, and their number was the highest in the adults. In all species, the pattern of the differing numbers of CR-immunoreactive neurons was similar. Scale bars: (Aa-Ia), $200 \mu \mathrm{m}$ and (Ab-Ic), $25 \mu \mathrm{m}$. (J) The percentage mean number of CR-immunoreactive neurons in whole layers in the somatosensory cortex. ${ }^{\mathrm{P}} \mathrm{P}<0.05$ vs. the young group; ${ }^{\mathrm{P}} \mathrm{P}<0.05$ vs. the adult group. Data are presented as the mean \pm the standard error of the mean (n=7/group). CR, calretinin.

\section{Discussion}

In the present study, the age-associated differences in the expression of $\mathrm{CB}, \mathrm{CR}$ and $\mathrm{PV}$ in the somatosensory cortex were examined in mice, rats and gerbils aged 1, 6 and 24 months using immunohistochemistry and western blotting. The number of CB-immunoreactive neurons was considerably higher in layers II and III when compared with the other layers, and the age-associated alterations in the total neuronal number was different among the species. In mice, the highest neuronal numbers were observed in the adult group and the lowest in the aged group. In the rats, the highest neuronal numbers were observed in the young group and the lowest in the aged group, and in the gerbils, the adults were observed to exhibit the highest neuronal numbers and the young gerbils the lowest. $\mathrm{Bu}$ et al (16) reported that the number of CB-immunoreactive neurons in whole layers (full cortical depth columns) of the primary visual cortex exhibited a consistent trend toward a decrease in the aged human brain. In addition, previous studies demonstrated a significant age-associated decrease in $\mathrm{CB}$ mRNA and CB immunoreactivity in the striatum $(13,25)$ and hippocampus $(30,31)$ of rodents during aging. These studies and the results of the present study indicate that $\mathrm{CB}$-immunoreactive interneurons in the rodent somatosensory cortex may be affected by age, and their numbers in the aged somatosensory cortex are decreased when compared with those in the adult. 


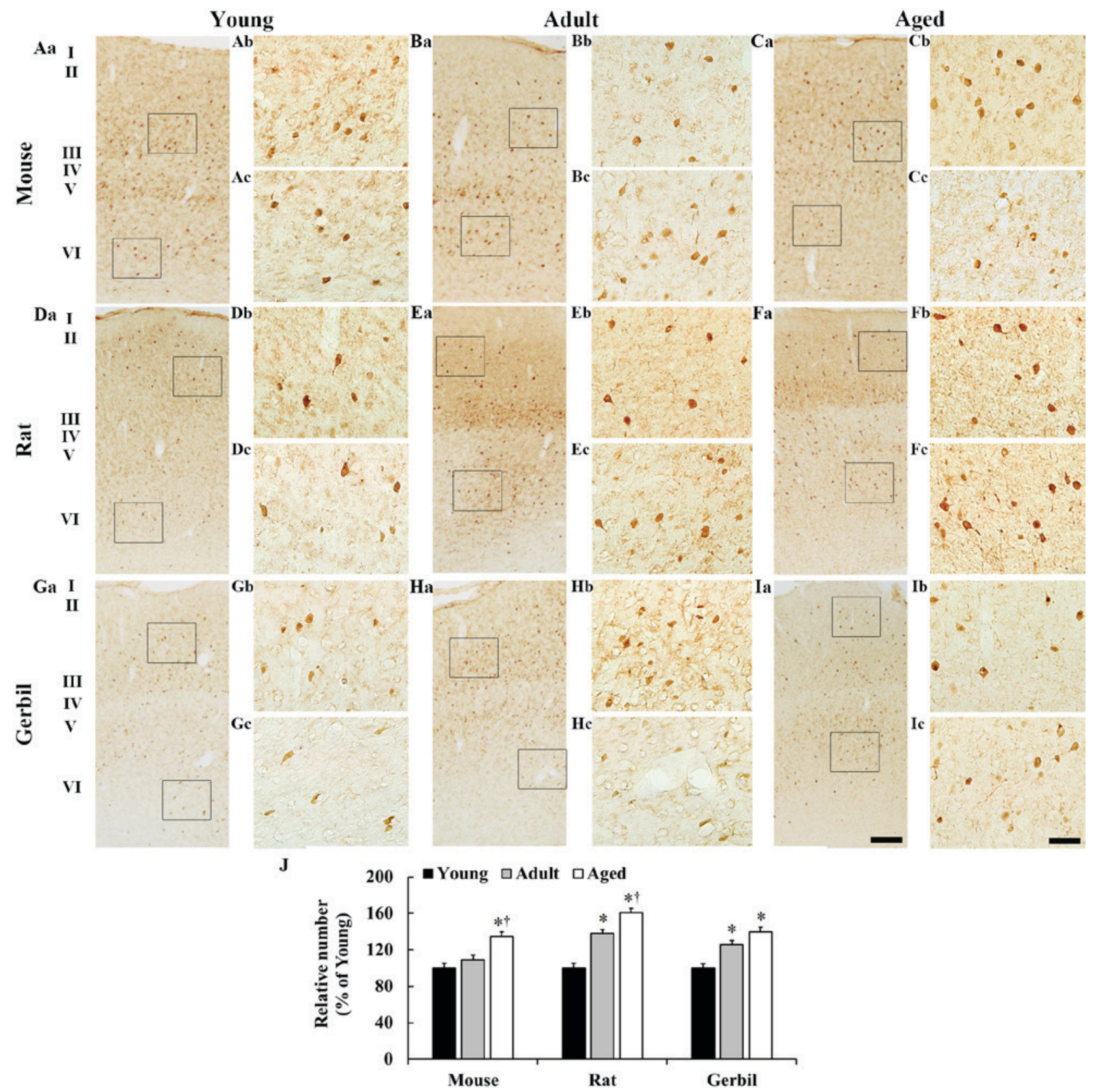

Figure 3. PV immunohistochemistry in the somatosensory cortex in the (A-C) mice, (D-F) rats and (G-I) gerbils. In all species, PV-immunoreactive neurons were primarily observed in layers II, III, V and VI. The number of PV-immunoreactive neurons increased with age. Scale bars: (Aa-Ia), $200 \mu \mathrm{m}$ and (Ab-Ic), $25 \mu \mathrm{m}$. (J) The percentage mean number of PV-immunoreactive neurons in whole layers in the somatosensory cortex. ${ }^{\mathrm{P}}<0.05 \mathrm{vs}$. the young group; ${ }^{\mathrm{T}} \mathrm{P}<0.05 \mathrm{vs}$. the adult group. Data are presented as the mean \pm the standard error of the mean ( $\mathrm{n}=7 /$ group). $\mathrm{PV}$, parvalbumin.

In the present study, the density of CR-immunoreactive neurons in the somatosensory cortex was markedly reduced when compared with CB- and PV-immunoreactive neurons, and CR-immunoreactive neurons were predominantly distributed in layers II and III. The pattern of age-associated change was similar amongst the three species, with their total numbers being increased in the adult animals and decreased in the aged animals. This finding is similar to a previous study, which revealed that the number of CR-immunoreactive neurons decreased significantly in temporal areas, including the auditory association cortex, middle temporal cortex and inferior temporal cortex in older human brains when compared with the young (16). In addition, it has been reported that the number of CR-immunoreactive neurons and $\mathrm{CR}$ protein levels were markedly decreased in the striatum (25) and hippocampus $(12,32)$ of aged rodents. These results, as well as those of the present study, indicate that CR expression in the rodent somatosensory cortex tends to decrease in the aged brain. In addition, the present study revealed that the number of CR-immunoreactive neurons in the young was significantly lower when compared with the adults.

The present study demonstrated that PV-immunoreactive neurons were primarily distributed in all layers except layer I. In addition, the number of PV-immunoreactive neurons in the somatosensory cortex increased with age and the highest numbers were observed in the aged group of all three species. A previous study demonstrated that the number of PV-immunoreactive neurons was not statistically 

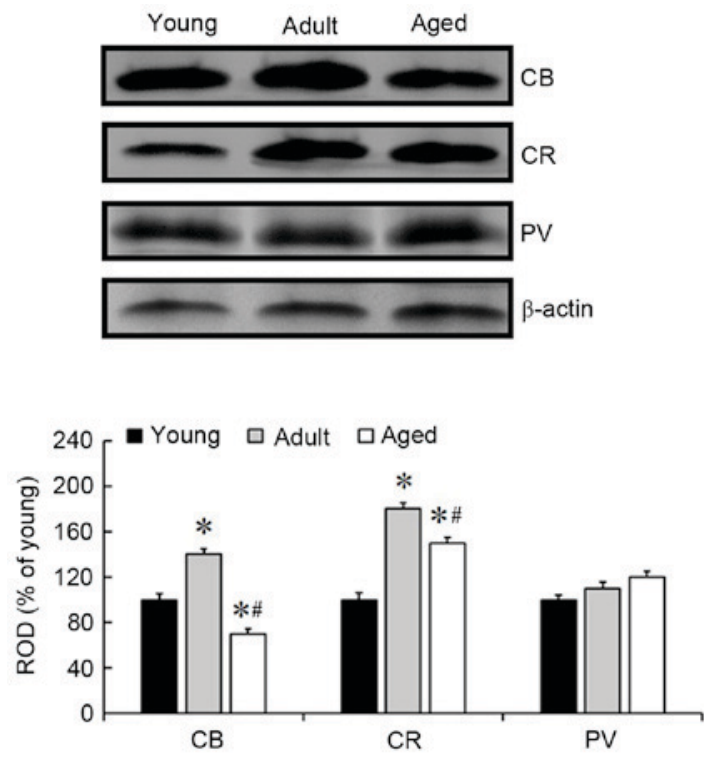

Figure 4. Western blot analysis of CB, CR and PV in the mouse cortex of the young, adult and aged groups. ROD is expressed as the percentage of the immunoblot band values in the young, adult and aged groups. Bands were normalized to $\beta$-actin expression. ${ }^{\text {}} \mathrm{P}<0.05$ vs. the young group; ${ }^{*} \mathrm{P}<0.05$ vs. the adult group. Data are presented as the mean \pm the standard error of the mean ( $n=5 /$ group). CB, calbindin-D28k; CR, calretinin; PV, parvalbumin; ROD, relative optical density.

different in various cortical areas of the aged human brain when compared with that of the young; however, they were marginally increased in the primary auditory cortex, prefrontal association cortex, premotor association cortex, middle temporal cortex and inferior temporal cortex (16). In addition, it has been reported that the number of $\mathrm{PV}$-immunoreactive neurons in the prefrontal cortex was not significantly different between young and aged canines (33). These results, as well as those of the present study, suggest that PV-immunoreactive GABAergic interneurons are relatively stable and resilient when compared with $\mathrm{CB}$ - and CR-immunoreactive neurons in the somatosensory cortex during the aging process.

In the present study, the distribution of CBP-containing neurons differed depending on the type of CBPs and the species. Previous studies have demonstrated that interneuron subtypes in the neocortex are involved in laminar or columnar inhibition (34) and that the axons of CBP-containing neurons generate different local circuits with pyramidal cells or GABAergic cells depending on the layer (35). In addition, GABAergic interneurons have exhibited electrophysiological differences in firing patterns; CB-positive neurons exhibited burst-spiking, CR-positive neurons displayed low-threshold spiking and $\mathrm{PV}$-expressed neurons presented fast-spiking firing properties $(3,4,6)$. Pugliese et al $(33)$ identified a specific vulnerability of CB-positive GABAergic interneurons in aged canines and the resistance of PV-immunoreactive neurons in aged canines with cognitive deficits. Therefore, taking these results into account, it is possible that the layer-specific, different patterns of age-associated alterations in CBP-containing neurons may be associated with each CBP-specific laminar distribution (different cellular composition form layer to layer) and their physiological differences.
For example, it has previously been demonstrated that visual cortical functions, including visual acuity, contrast sensitivity and orientation sensitivity deteriorate in the elderly (36-38). In addition, the electrophoretic application of GABA or the GABA agonist may restore orientation turning in visual cortical neurons in monkeys (39). Therefore, age-associated reductions of intra cortical inhibition in the somatosensory cortex may contribute to a decline in somatosensory functions (5).

In conclusion, the results of the present study demonstrate that the number of CBP-immunoreactive neurons in the rodent somatosensory cortex, which displayed different neuronal populations and laminar distribution, were markedly altered with normal aging. The CB and CR types exhibited a tendency to decrease, while PV displayed a stable pattern. These results indicate that CB- and CR-immunoreactive neurons are altered in the rodent somatosensory cortex, and present one feature of general age-associated processes.

\section{Acknowledgements}

The present study was supported by the Bio \& Medical Technology Development Program of the NRF funded by the Korean government, MSIP (Ministry of Science, ICT and Future Planning; grant no. NRF-2015M3A9B6066835) and by a Priority Research Centers Program grant (grant no. NRF-2009-0093812) through the National Research Foundation of Korea funded by the Ministry of Science, ICT and Future Planning.

\section{References}

1. Aronoff R and Petersen CC: Layer, column and cell-type specific genetic manipulation in mouse barrel cortex. Front Neurosci 2: 64-71, 2008.

2. DeFelipe J and Fariñas I: The pyramidal neuron of the cerebral cortex: Morphological and chemical characteristics of the synaptic inputs. Prog Neurobiol 39: 563-607, 1992.

3. Kawaguchi Y and Kubota Y: GABAergic cell subtypes and their synaptic connections in rat frontal cortex. Cerebral Cortex 7: 476-486, 1997.

4. Hioki H: Compartmental organization of synaptic inputs to parvalbumin-expressing GABAergic neurons in mouse primary somatosensory cortex. Anat Sci Int 90: 7-21, 2015.

5. Lehmann K, Steinecke A and Bolz J: GABA through the ages: Regulation of cortical function and plasticity by inhibitory interneurons. Neural Plast 2012: 892784, 2012.

6. Kawaguchi Y, Wilson CJ, Augood SJ and Emson PC: Striatal interneurones: Chemical, physiological and morphological characterization. Trends Neurosci 18: 527-535, 1995.

7. Baimbridge KG, Celio MR and Rogers JH: Calcium-binding proteins in the nervous system. Trends Neurosci 15: 303-308, 1992.

8. Burgoyne RD: Neuronal calcium sensor proteins: Generating diversity in neuronal $\mathrm{Ca} 2+$ signalling. Nat Rev Neurosci 8: 182-193, 2007.

9. Arundine $\mathrm{M}$ and Tymianski M: Molecular mechanisms of calcium-dependent neurodegeneration in excitotoxicity. Cell Calcium 34: 325-337, 2003.

10. Oh MM, Oliveira FA, Waters J and Disterhoft JF: Altered calcium metabolism in aging CA1 hippocampal pyramidal neurons. J Neurosci 33: 7905-7911, 2013.

11. Kuchibhotla KV, Goldman ST, Lattarulo CR, Wu HY, Hyman BT and Bacskai BJ: Abeta plaques lead to aberrant regulation of calcium homeostasis in vivo resulting in structural and functional disruption of neuronal networks. Neuron 59: 214-225, 2008.

12. Villa A, Podini P, Panzeri MC, Racchetti G and Meldolesi J: Cytosolic $\mathrm{Ca} 2+$ binding proteins during rat brain ageing: Loss of calbindin and calretinin in the hippocampus, with no change in the cerebellum. Eur J Neurosci 6: 1491-1499, 1994. 
13. Kishimoto J, Tsuchiya T, Cox H, Emson PC and Nakayama Y: Age-related changes of calbindin-D28k, calretinin and parvalbumin mRNAs in the hamster brain. Neurobiol Aging 19: 77-82, 1998.

14. Gonchar Y, Wang Q and Burkhalter A: Multiple distinct subtypes of GABAergic neurons in mouse visual cortex identified by triple immunostaining. Front Neuroanat 1: 3, 2008.

15. Litwinowicz B, Labuda C, Kowiański P, Spodnik JH, Ludkiewicz B, Wójcik S and Moryś J: Developmental pattern of calbindin D28k protein expression in the rat striatum and cerebral cortex. Folia Morphol (Warsz) 62: 327-329, 2003.

16. Bu J, Sathyendra V, Nagykery N and Geula C: Age-related changes in calbindin-D28k, calretinin, and parvalbumin-immunoreactive neurons in the human cerebral cortex. Exp Neurol 182: 220-231, 2003.

17. Selakovic V, Rauš Balind S, Radenovic L, Prolić Z and Janać B: Age-dependent effects of ELF-MF on oxidative stress in the brain of Mongolian gerbils. Cell Biochem Biophys 66: 513-521, 2013.

18. Lores-Arnaiz S, Lombardi P, Karadayian AG, Orgambide F Cicerchia D and Bustamante J: Brain cortex mitochondrial bioenergetics in synaptosomes and non-synaptic mitochondria during aging. Neurochem Res 41: 353-363, 2016.

19. Zheng T, Lv Q, Lei X, Yin X and Zhang B: Spatial distribution of 5-hydroxymethyl cytosine in rat brain and temporal distribution in striatum. Neurochem Res 40: 688-697, 2015.

20. Vanhooren $\mathrm{V}$ and Libert $\mathrm{C}$ : The mouse as a model organism in aging research: Usefulness, pitfalls and possibilities. Ageing Res Rev 12: 8-21, 2013.

21. Quinn R: Comparing rat's to human's age: How old is my rat in people years? Nutrition 21: 775-777, 2005.

22. Demetrius L: Aging in mouse and human systems. Ann N Y Acad Sci 1067: 66-82, 2006

23. Gorbunova V, Bozzella MJ and Seluanov A: Rodents for comparative aging studies: From mice to beavers. Age (Dordr) 30: 111-119, 2008

24. Institute of Laboratory Animal Research, Committee for the Update of the Guide for the Care and Use of Laboratory Animals, National Research Council. Guide for the care and use of laboratory animals. 8th edition. Washington, (DC), National Academies Press, pp220, 2011.

25. Bae EJ, Chen BH, Shin BN, Cho JH, Kim IH, Park JH, Lee JC, Tae HJ, Choi SY, Kim JD, et al: Comparison of immunoreactivities of calbindin-D28k, calretinin and parvalbumin in the striatum between young, adult and aged mice, rats and gerbils Neurochem Res 40: 864-872, 2015.
26. Keith BJ and Franklin George P: The Mouse Brain in Stereotaxic Coordinates. San Diego, California:Academic Press, Inc., pp20-39, 1997.

27. George P and Charles W: The Rat Brain in Stereotaxic Coordinates. 2nd edition. San Diego, California, Academic Press, Inc., pp11-24, 1986.

28. William JL, Peter L and Anthony MV: A stereotaxic Atlas of the Mongolian Gerbil Brain (Meriones unguiculatus). Ann Arbor, Michigan, Ann Arbor Science Publishers Inc., pp36-60, 1974.

29. Lee JC, Ahn JH, Lee DH, Yan BC, Park JH, Kim IH, Cho GS, Kim YM, Lee B, Park CW, et al: Neuronal damage and gliosis in the somatosensory cortex induced by various durations of transient cerebral ischemia in gerbils. Brain Res 1510: 78-88, 2013.

30. Shetty AK and Turner DA: Hippocampal interneurons expressing glutamic acid decarboxylase and calcium-binding proteins decrease with aging in Fischer 344 rats. J Comp Neurol 394: 252-269, 1998 .

31. Lee CH, Hwang IK, Yoo KY, Choi JH, Park OK, Lee JC, Jeong YG, Lee IS and Won MH: Calbindin d-28k immunoreactivity and its protein level in hippocampal subregions during normal aging in gerbils. Cell Mol Neurobiol 29: 665-672, 2009.

32. Lee CH, Hwang IK, Choi JH, Yoo KY, Park OK, Huh SO, Lee YL, Shin HC and Won MH: Age-dependent changes in calretinin immunoreactivity and its protein level in the gerbil hippocampus. Neurochem Res 35: 122-129, 2010.

33. Pugliese M, Carrasco J, Geloso MC, Mascort J, Michetti F and Mahy $\mathrm{N}$ : $\gamma$-aminobutyric acidergic interneuron vulnerability to aging in canine prefrontal cortex. J Neurosci Res 77: 913-920, 2004.

34. DeFelipe J and Fariñas I: The pyramidal neuron of the cerebral cortex: Morphological and chemical characteristics of the synaptic inputs. Prog Neurobiol 39: 563-607, 1992.

35. Meskenaite V: Calretinin-immunoreactive local circuit neurons in area 17 of the cynomolgus monkey, Macaca fascicularis. J Comp Neurol 379: 113-132, 1997.

36. Ferrer-Blasco T, González-Méijome JM and Montés-Micó R: Age-related changes in the human visual system and prevalence of refractive conditions in patients attending an eye clinic. J Cataract Refract Surg 34: 424-432, 2008

37. Lehmann K, Schmidt KF and Löwel S: Vision and visual plasticity in ageing mice. Restor Neurol Neurosci 30: 161-178, 2012.

38. Hua T, Li X, He L, Zhou Y, Wang Y and Leventhal AG: Functional degradation of visual cortical cells in old cats. Neurobiol Aging 27: 155-162, 2006.

39. Leventhal AG, Wang Y, Pu M, Zhou Y and Ma Y: GABA and its agonists improved visual cortical function in senescent monkeys. Science 300: 812-815, 2003. 